\title{
Contextual Factors Influencing Physicians' Perception of Antibiotic Prescribing in Primary Care in Germany - A Prospective Observational Study
}

Annika Queder ( $\sim$ Annika.Queder@med.uni-heidelberg.de )

University Hospital Heidelberg

Christine Arnold

University Hospital Heidelberg

Michel Wensing

University Hospital Heidelberg

Regina Poß-Doering

University Hospital Heidelberg

\section{Research Article}

Keywords: rational antibiotic use, primary care, physicians' decision-making, context analysis

Posted Date: July 27th, 2021

DOI: https://doi.org/10.21203/rs.3.rs-674135/v2

License: (a) (i) This work is licensed under a Creative Commons Attribution 4.0 International License.

Read Full License

Version of Record: A version of this preprint was published at BMC Health Services Research on March 12th, 2022. See the published version at https://doi.org/10.1186/s12913-022-07701-3. 


\section{Abstract}

Background: Antimicrobial resistance is a worldwide challenge for health services and systems alike. To reduce the overuse of antibiotics, multifaceted interventions are often used to achieve sustainable effects. It can be assumed that these effects are influenced by contextual factors. Embedded in the cluster randomized trial ARena (Sustainable reduction of antibiotic-induced antimicrobial resistance), the aim of this present study was to identify contextual factors associated with practitioners' perceptions of antibiotic prescribing in German primary health care.

Methods: In a prospective observational study, data were generated in a three-wave survey study between January 2018 and July 2019. Analysis was performed using logistic regression models. The outcome of interest was the physician perceived impact of participating in the ARena project on decision-making regarding antibiotic prescribing, the independent variables of interest included individual characteristics, intervention arm allocation, primary care network (PCN) environment and characteristics of the medical practice.

Results: $46.8 \%(n=126)$ of participants indicated to have perceived an impact on their decision-making regarding antibiotic prescribing by participating in the ARena project. Bivariate logistic regression analyses indicated that work experience (odds ratio (OR) 1.05, 95\% confidence interval (Cl) 1.006-1.103), PCN environment (OR 2.06, 95\% Cl 1.256-3.363), structural conditions (OR 1.66, 95\% Cl 1.161-2.371), environment of existing processes (OR 1.46, 95\% $\mathrm{Cl} 1.011-2.094)$, and externally defined general conditions (OR 1.57, 95\% Cl 1.035-2.378) were associated with physicians' perceived impact of participating in the ARena project on decision-making regarding antibiotic prescribing. In the multivariate logistic regression analysis, only work experience OR 1.05 (95\% Cl 1.001-1.104) continuously showed a significant influence.

Conclusions: This study indicates that contextual factors at individual, practice, and system level influence physicians' perceptions of antibiotic prescribing.

Trial registration: ISRCTN, ISRCTN58150046 (registered 13.09.2017).

\section{Background}

Antimicrobial resistance (AMR) is a worldwide challenge for health services and systems alike. In order to meet the challenge AMR poses for the general public and the health care system in Germany, the policy to curb the prescribing of antibiotics in human medicine has been strengthened in Germany by a national political agenda [1].

The AMR rate is strongly correlated with the use of antibiotics in human medicine [1, 2]. A major proportion of antibiotic use in human health care is related to the primary health care sector, which reinforces that it is an important area for research and action $[2,3]$. 
In Germany, the primary care sector accounts for a large proportion of antibiotic consumption [1]. The use of antibiotics in Germany decreased from 13.4 defined daily dose (DDD) per 1,000 inhabitants per day in 2010 to 11.4 DDD per 1,000 inhabitants per day in 2019 [4]. Although antibiotic consumption in Germany is considered moderate compared to other European countries, there is still considerable potential for improvement $[1,5]$.

To meet these challenges, the ARena (Sustainable reduction of antibiotic-induced antimicrobial resistance) project was initiated to promote the rational and appropriate use of antibiotics for acute, noncomplicated and self-limiting infections in primary care. Aiming to promote awareness and understanding of the growing challenges associated with AMR, ARena addressed knowledge and attitudes about the use of antibiotics of primary care physicians, primary care teams as well as patients, using a multifaceted strategy with multiple interacting intervention components [6]. Complex multiple component interventions $(\mathrm{CMCl})$ are increasingly used in health services research. As results, a synergy of various effects being able to address the high complexity in health care and the fast progression of innovation are expected $[7,8]$. Particular interest is paid to intervention- and context-dependent factors that might cause changes [7].

Even though the concept of context is widely used in health services research, a consistent definition and an application in practice still lacks [9]. In the present study, the term 'context' is understood as defined by Pfadenhauer et al. [10] "reflects a set of characteristics and circumstances [...] within which the implementation is embedded" and "interacts, influences, modifies and facilitates or constrains the intervention and its implementation" [10]. Therefore context is an overarching concept that encompasses physical location as well as relationships, roles and interactions at multiple levels [10]. In the Context and Implementation of Complex Interventions (CICl) framework, context comprises seven domains: (1) epidemiological, (2) geographical, (3) ethical, (4) socio-cultural, (5) socio-economic, (6) legal, and (7) political context [10]. Therefore, it can be assumed that these contextual factors have a significant impact on a desired change in behavior. To understand which factors influence practitioners' antibiotic prescribing patterns, it is important to understand how behavior occurs. The Theory of Planned Behavior (TPB) describes four central aspects to explain behavior: (1) attitude towards behavior, (2) subjective norms related to behavior, and (3) perceived behavioral control. These predict (4) behavioral intentions which in turn can lead to behavior [11].

The aim of this study was to identify contextual factors relevant to physicians' decision-making in antibiotic prescribing in German primary health care. Data from the survey study conducted within the ARena process evaluation were used to explore whether primary care physicians perceived their participation in the ARena project to have an impact on their decision-making in antibiotic prescribing.

Potential findings are useful for implementation science to identify determinants and understanding mechanisms of action in order to achieve the uptake of research findings into routine health care, thus improving health care services [7]. 


\section{Methods}

To report this study, the "Strengthening the Reporting of Observational Studies in Epidemiology (STROBE-) Statement" checklist was used [12].

\section{Research design and conceptual framework}

For this study, a prospective observational study design following an exploratory approach was used. ARena was a cluster randomized trial conducted in 14 primary care networks (PCNs) in Bavaria and North-Rhine Westphalia with the aim of a sustainable reduction of antibiotic use in acute, noncomplicated and self-limiting infections. Using a multifaceted strategy with multiple interacting intervention components, primary care physicians and teams in the three intervention arms (I, II, III) received intervention components containing e-learning on communication, quality circles, performancebased additional reimbursement, public information campaigns, and culture-sensitive information material for patients in printed format. Arm II received additional culture-sensitive information material for patients in digital format via tablet computers provided in the practices' waiting areas. Intervention arm III received a computerized decision support system and additional quality circles. Randomization for the intervention study was done by statisticians at the Department of Medical Biometry, Institute of Medical Biometry and Informatics, University Hospital Heidelberg. The evaluation research cover both an outcome and a process evaluation. Detailed descriptions of the project [6] and the process evaluation were published elsewhere [13].

Data generated through the three-wave survey study within the ARena process evaluation were used. The study-specific survey questionnaires (T0, T1, T2) were developed based on the TPB [11]. The TPB as well as the $\mathrm{CICl}$ framework, further scientific literature and specific factors derived from the project

environment (such as membership in a PCN or the assignment to one of the intervention arms) were used to identify possible contextual factors underlying the analysis of this study.

\section{Data collection}

Data were collected by the study team at the Department of General Practice and Health Services Research at the University Hospital Heidelberg at three measurement points in January 2018 (T0), October 2018 (T1), and July 2019 (T2). All primary care physicians participating in the ARena project were invited to participate in the survey $(T 0 n=303, T 1 n=312, T 2 n=292)$. To increase the response rates, E-mail reminders were sent out after four weeks.

Survey questionnaires comprised socio-demographic questions, items focused on implemented intervention components, and relevant contextual factors as well as prescribing decisions and general perceptions regarding antibiotics. An overview of all relevant questions for this analysis is provided in Additional file 1. For analysis, data from all completed questionnaires were digitalized and transferred into IBM SPSS Statistics 24. Findings are reported here with a focus on contextual factors of the ARena project and their impact on decision-making in antibiotic prescribing by primary care providers. 


\section{Outcome}

The physician's perceived impact of participation in the ARena project on decision-making regarding antibiotic prescribing was measured with a 5-point Likert-Scale (completely

disagree/disagree/neutral/agree/completely agree) in T2. For bivariate and binary logistic regression analysis, the ordinal variable was modified to a dichotomy variable: ' 0 ' = participation in the ARena project did not impact decision-making in antibiotic prescribing (completely disagree/disagree/neutral) and ' 1 ' = participation in the ARena project did impact the decision-making in antibiotic prescribing (agree/ completely agree). The value 'neutral' indicated that participants were indecisive or could not attribute a clear impact of their study participation on their decision-making regarding antibiotics prescribing.

\section{Predictive factors}

Possible influencing factors were examined based on the theoretical frameworks of the TPB and $\mathrm{CICl}$, prior study findings and specific factors derived from the project environment. In this study, four domains of possible influencing factors were included: (I) individual characteristics, (II) intervention arm allocation, (III) PCN environment, and (IV) general characteristics of the medical practice.

(I): All variables of individual characteristics were measured at time of T0. Individual-level factors included in this study were sex (female or male), age and work experience (count variables), employment type (employed or independent), employment time (part time or full time), and medical specialty (free text response variable classified as dichotomous variable of 'general practitioner or specialist').

(II): To investigate the impact of the interventions on decision-making in antibiotic prescribing, the membership in the intervention arm was tested. The assignment to the intervention arm was measured in a categorial variable with the values I, II and III.

(III): The context of PCN can be assigned to both, the socio-cultural and ethical context domain of the $\mathrm{CICl}$ and was measured with eleven 5-Point Likert-Scale items in T0 (completely disagree to completely agree). For this study, the respective mean value scores of the items were formed for all completely answered cases. The scale was validated with a Cronbach's a coefficient of 0.898 (see Additional file 2). The mean value score was interpreted as a scale from 1 (not supportive) to 5 (very supportive), indicating how supportive participants consider their PCNs' environment.

(IV): General conditions of medical practice were described by the number of patients per quarter of year classified as group $1(<500)$, group $2(500-1,000)$ group $3(1,001-1,500)$ and group $4(>1,500)$ and were re-coded in a dichotomous variable indicating practice size with group $1(\leq 1,000)$ and group $2(\geq 1,001)$ patients per quarter of year for this analysis. Practice area was classified by five categories: group 1 (< $5,000)$, group $2(5,000-<20,000)$, group $3(20,000-<100,000)$, group $4(100,000-<500,000)$, group 5 $(\geq 500,000)$ inhabitants and included in this analysis as a dichotomous variable $(<100,000$ and $\geq$ $100,000)$ population. These factors are placed in the epidemiological and geographic context domains of 
the $\mathrm{CICl}$. Further contextual factors such as "structural conditions", "environment of existing processes in the practice" and "externally defined general conditions" were each recorded with six 5-Point Likert-Scale items (completely disagree to completely agree). Mean value scores were calculated (scale was validated with a Cronbach's a coefficient of $0.921 / 0.907 / 0.890$ ) for the respective domains as a scale that indicates how supportive each context-domain is perceived by the participants (scale from 1 (not supportive) to 5 (very supportive)). For detailed survey items see Additional file 2 . All items in this domain were measured in $\mathrm{TO}$. In relation to the $\mathrm{CICl}$, structural conditions are to be classified in the domains of the geographical and socio-economic context. The environment of existing processes in the medical practice is linked to the geographical and socio-cultural context domain, and externally defined general conditions are to be classified to the socio-economic, political and legal context domain of the $\mathrm{CICl}$.

\section{Statistical analysis}

IBM SPSS Statistics 24 (IBM, Armonk, NY, USA) was used for all analyses. Data visualization was performed using Microsoft Excel 2016 (Microsoft Corp., Redmond, WA, USA).

All variables were analyzed descriptively (absolute and relative frequencies for nominal scale levels and means with Standard Deviations (SDs) for continuous variables). Respondents' baseline characteristics (T0) were summarized and a comparison between intervention arms was made using adequate tests depending on the type of variables (Phi/Cramer-V, Chi ${ }^{2}$, Eta coefficient, and Kruskal-Wallis (H-)Test for comparison of mean values).

Bivariate analyses were performed using cases answered at T0 and T2 between the dependent variable (measured in T2) and 10 predictive variables (measured in T0). Correlation tests (Cramer-V and Eta) and binary logistic regression models were performed to evaluate correlations between the predictive variables and the dependent variable. The Odds Ratio (OR) was calculated with a Confidence Interval (Cl) of $95 \%$.

Due to the small underlying number of cases $(n=152)$, the inclusion of independent variables was limited [14]. Thus, to take the theoretical background of the $\mathrm{CICl}$ into account, four binary logistic regression models were performed separately to consider the influence of the predictive contextual factors in the four domains (1) individual characteristics, (2) intervention arm allocation, (3) PCN environment, and (4) general characteristics of the medical practice. Only statistically significant results ( $p$-value $\leq 0.05)$ were included in the multivariate analyses. A multivariate binary logistic regression model was employed to analyze the influence of the contextual factors on the perceived impact of participation in the ARena project on decision-making regarding antibiotic prescribing. The predictive factors were included stepwise to consider changes in coefficients.

The requirements for binary logistic regression were examined and multi-collinearity was tested. Since there was a high and statistically significant correlation between age and work experience of participants (Pearson's $r=0.913, p=<0.001$ ), the variable 'physician age' was excluded as previous research found 
work experience as an important factor that influences the decision-making of antibiotic prescribing [3, 15].

The participants were nested in a total of 14 PCNs (2-27 per network). In order to take the clustered structure of the data into account, a Multilevel Analysis (MLA) was carried out for the PCNs. MLA is a method used in health services research to examine the associations between context in health care organizations and the objective $[16,17]$. Therefore, the intra-cluster correlation coefficient (ICC) in multilevel logistic regression was calculated to examine the share of the between-cluster variance in the total variance [18]. Finally, a mixed logistic model with a random intercept defined by any given PCN was employed.

Missing values were analyzed descriptively and a Little's Missing Completely at Random (MCAR)-Test was performed $(p=0.185)$, showing that missing values were completely at random (see Additional file $3)$. The significance level for all analyses was set at $5 \%$.

\section{Results}

\section{Sample characteristics}

Response rates were 75.5\% (T0 $n=229), 64 \%(\mathrm{~T} 1 n=200)$ and 63.3\% (T2 $n=184)$ [19]. At time of T0, 148 $(55 \%)$ of all responders were male and the average age was 54 years (SD 7.59). 132 (49.1\%) were general practitioners (GPs) and 95 (35.3\%) were specialized physicians, including qualifications of internal medicine, otolaryngology, urology, paediatrics, pneumology and gynaecology. On average, participants had 25.5 years (SD 7.94) of work experience. 65 (24.2\%) of them worked in a single practice, 154 (57.2\%) in group practices, and $10(3.7 \%)$ in shared rooms. 193 (71.7\%) were self-employed. 90 (33.5\%) of the participants worked full time. Table 1 shows the characteristics of the participating physicians at T0, differentiated according to the intervention arms. There were no statistically significant differences in relevant characteristics of participants between the intervention arms. 
Table 1

Characteristics of participants by intervention arm allocation (T0)

\begin{tabular}{|lllll|}
\hline Characteristics & Total & Intervention arm I & Intervention arm II & Intervention arm III \\
& $\mathbf{N = 2 2 9}$ & $\mathbf{n = 7 6 ( 3 3 . 1 \% )}$ & $\mathbf{n = 7 3 ( 3 2 \% )}$ & $\mathbf{n = 8 0}(\mathbf{3 4 . 9 \% )}$ \\
\hline Sex & $224(100)$ & $73(100)$ & $73(100)$ & $78(100)$ \\
\hline Female, $\mathrm{n}(\%)$ & $76(33.9)$ & $18(24.7)$ & $25(34.2)$ & $33(42.3)$ \\
\hline Male, $\mathrm{n}(\%)$ & $148(66.1)$ & $55(75.3)$ & $48(65.8)$ & $45(57.7)$ \\
\hline Age mean (SD) & $54.4(7.6)$ & $54.7(7.5)$ & $54.6(8.3)$ & $54.1(7.1)$ \\
\hline Medical Speciality & $227(100)$ & $76(100)$ & $71(100)$ & $80(100)$ \\
\hline GP, $\mathrm{n}$ (\%) & $132(58.1)$ & $44(57.9)$ & $40(56.3)$ & $48(60.0)$ \\
\hline Specialists, n (\%) & $95(41.9)$ & $32(42.1)$ & $31(43.7)$ & $32(40.0)$ \\
\hline Work experience, & & & $24.9(8)$ \\
\hline in years, mean (SD) & $25.5(7.9)$ & $25.8(7.3)$ & $25.8(8.6)$ & \\
\hline GP = General Practitioner; SD = Standard deviation & & \\
\hline
\end{tabular}

\section{Outcome and contextual factors}

With 126 (46.8\%) physicians, nearly half of participants indicated to have perceived an impact on their decision-making in antibiotic prescribing by partaking in the ARena project (T2). This estimation was homogeneous across all three intervention arms $\left(\mathrm{Chi}^{2}(2): 0.076, p=0.963\right)$.

The PCN as a contextual factor of the ARena project was assessed as an overall supportive environment with a mean value of 3.93 (SD 0.73, $n=224$ ). Regarding the intervention arms I, II and III, group comparisons showed statistically significant differences between the intervention arms regarding the evaluation of the PCN environment (H-Test: 6.499(2), $p=0.039$ ). With a mean value of 4.10 (SD 0.71, $n=$ 60 ), intervention arm II rated their PCN environment as more supportive than intervention arm I (mean: 3.83 , SD 0.70, $n=53$ ) and intervention arm III (mean: 3.86, SD 0.76, $n=71$ ).

Considering the context of the medical practice, most of the respondents worked in catchment areas of less than 100,000 population $(52.4 \%, n=141)$ and about $32 \%(n=86)$ in areas of more than 100,000 population. Group comparisons showed statistically significant differences regarding the practice area (Chi $\left.{ }^{2}(2): 66.251, p<0.001\right)$. There were no significant differences between the intervention arms in other contextual factors (see Additional file 4$)$. About $13 \%(n=34)$ of the participating physicians indicated to treat less than 1,000 patients and $67 \%(n=180)$ to treat more than 1,000 patients per quarter of a year.

While structural conditions (mean: 2.9, SD 1.1) as well as externally defined general conditions (mean 2.67, SD 0.93 ) of the practice were assessed as a less supportive environment, existing processes and the 
organisation of the practice as a whole were shown to be considered a supportive environment (mean: 3.3, SD 1.0).

\section{Relationship between decision-making and contextual factors}

Bivariate results refer to the number of participants who returned the survey questionnaires at T0 and T2 $(n=152)$. Group comparison showed that these participants did not differ significantly from other participants.

A total of 5 of 10 predictors showed significant bivariate association with the outcome.

Table 2 shows statistically significant relationships between the perceived impact of participation in the ARena project on decision-making in antibiotic prescribing and work experience (Eta: 0.189, $p=0.026$ ), PCN environment (Eta: $0.244, p=0.003$ ), structural conditions (Eta: $0.235, p=0.004$ ), environment of existing processes (Eta: $0.167, p=0.004$ ), and externally defined general conditions (Eta: $0.176, p=$ 0.032). 
Table 2

Bivariate analyses of contextual factors and the dependent variable (PIP-ARena)

\begin{tabular}{|c|c|c|c|c|}
\hline Variables & $\begin{array}{l}\text { PIP- } \\
\text { ARena } \\
n=108 \\
n(\%)\end{array}$ & $\begin{array}{l}\text { No PIP-ARena } \\
n=43 \\
n(\%)\end{array}$ & Correlation & $P$ value \\
\hline \multicolumn{5}{|l|}{ Individual characteristics } \\
\hline Sex & $107(72.3)$ & $41(27.7)$ & $0.046^{a}$ & 0.578 \\
\hline female & $34(69.4)$ & 15 (30.6) & & \\
\hline male & 73 (73.7) & $26(26.3)$ & & \\
\hline Medical Specialty & $107(71.8)$ & $42(28.2)$ & $0.063^{a}$ & 0.438 \\
\hline GP & 66 (74.2) & $23(25.8)$ & & \\
\hline Specialists & $41(68.3)$ & $19(31.7)$ & & \\
\hline Work experience, mean SD & $27(8)$ & $24(7)$ & $0.189^{b}$ & 0.026 \\
\hline Intervention arm allocation & $108(71.5)$ & $43(28.5)$ & $0.108^{a}$ & 0.414 \\
\hline I & $39(78.0)$ & $11(22.0)$ & & \\
\hline ॥ & $40(70.2)$ & $17(29.8)$ & & \\
\hline III & $29(65.9)$ & $15(34.1)$ & & \\
\hline PCN environment mean SD & $4.08(0.7)$ & $3.67(0.8)$ & $0.244^{b}$ & 0.003 \\
\hline \multicolumn{5}{|l|}{ General characteristics of medical practice } \\
\hline Number of patients per quarter of year & $103(74.1)$ & $36(25.9)$ & $0.042^{a}$ & 0.618 \\
\hline$<1,000$ patients & 18 (78.3) & $5(21.7)$ & & \\
\hline$>1,000$ patients & $85(73.3)$ & 31 (26.7) & & \\
\hline Practice area & $107(71.8)$ & $42(28.2)$ & $0.020^{a}$ & 0.841 \\
\hline$<100,000$ population & $71(72.4)$ & 27 (27.6) & & \\
\hline$\geq 100,000$ population & 36 (70.6) & $15(29.4)$ & & \\
\hline
\end{tabular}

$\mathbf{a}=$ CramerV; $^{\mathbf{b}}=$ Eta

PIP-ARena = perceived impact of participation in the ARena project on the decision-making regarding antibiotic prescribing; GP = General Practitioner; $\mathrm{PCN}$ = primary care network; $\mathrm{SD}$ = Standard deviation 


\begin{tabular}{|c|c|c|c|c|}
\hline Variables & $\begin{array}{l}\text { PIP- } \\
\text { ARena } \\
n=108 \\
n(\%)\end{array}$ & $\begin{array}{l}\text { No PIP-ARena } \\
n=43 \\
n(\%)\end{array}$ & Correlation & $P$ value \\
\hline Structural conditions, mean SD & $3.06(1.1)$ & $2.49(1.0)$ & $0.235^{b}$ & 0.004 \\
\hline Environment of existing processes, mean SD & $3.38(1.0)$ & $3.02(1.1)$ & $0.167^{b}$ & 0.004 \\
\hline $\begin{array}{l}\text { External defined general conditions, mean } \\
\text { SD }\end{array}$ & $2.76(0.9)$ & $2.41(0.8)$ & $0.176^{b}$ & 0.032 \\
\hline \multicolumn{5}{|l|}{$\mathbf{a}=$ CramerV $^{\mathbf{b}}=\mathrm{Eta}$} \\
\hline
\end{tabular}

No statistically significant relationship was observed between the outcome measure and sex, the qualification of physicians, the intervention arm allocation, the number of patients per quarter of year, or the practice area.

\section{Influence on the perceived impact of participation in the ARena project on decision-making regarding antibiotic prescribing}

The number of cases limited the inclusion of predictive factors in the multivariate logistic regression model. Thus, only statistically significant predictors in the bivariate analyses were included for further analyses. First, all factors were examined in bivariate logistic regression models (Table 3).

Table 3

Bivariate logistic regression analyses of contextual factors influencing PIP-ARena

\begin{tabular}{|c|c|c|c|c|c|c|c|}
\hline Variables & B & SE & Wald & df & $P$ value & OR & $\mathrm{Cl} 95 \%$ \\
\hline Experience year & 0.05 & 0.02 & 4.82 & 1 & 0.028 & 1.05 & $1.006-1.103$ \\
\hline PCN environment & 0.72 & 0.25 & 8.22 & 1 & 0.004 & 2.06 & $1.256-3.363$ \\
\hline Structural conditions & 0.50 & 0.18 & 7.74 & 1 & 0.005 & 1.66 & $1.161-2.371$ \\
\hline Environment of existing processes & 0.37 & 0.19 & 4.08 & 1 & 0.043 & 1.46 & $1.011-2.094$ \\
\hline External defined general conditions & 0.45 & 0.21 & 4.51 & 1 & 0.034 & 1.57 & $1.035-2.378$ \\
\hline
\end{tabular}

The bivariate logistic regression analyses showed that both the models as a whole and the individual coefficients of the contextual factors were significant. Nagelkerkes $R^{2}$ was between 0.04 and 0.08 . This resulted in a Cohen effect strength of $0.20-0.30$ which corresponds to a medium effect [20]. 
Practitioners with longer work experience were more likely to perceive an impact on decision-making regarding antibiotic prescribing by participating in the ARena project (OR 1.05, 95\% Cl 1.006-1.103). OR 2.06 (95\% Cl 1.256-3.369) was identified for PCN environment. Thus, the more supportive the PCN environment was perceived to be, the more likely participation in the ARena project was to influence decision-making regarding antibiotic prescribing. Higher perceived support from structural conditions (OR $1.66,95 \% \mathrm{Cl} 1.161-2.371$ ), existing processes (OR 1.46, 95\% $\mathrm{Cl} 1.011-2.094$ ), and externally defined general conditions (OR $1.57,95 \% \mathrm{Cl} 1.035-2.378)$ increased the relative probability of influencing decision-making in antibiotic prescription through participation in the ARena project by $66 \%, 46 \%$, and $57 \%$ respectively.

Subsequently, all factors were included stepwise in a multivariate model. The sample size in the hierarchical logistic regression analysis was $n=142$, as all variables were present. The model was statistically significant $\left(\mathrm{Chi}^{2}(5)=16.128, p=0.006\right)$. Nagelkerkes $\mathrm{R}^{2}$ was at 0.15 . This resulted in a Cohen effect strength of 0.43 which corresponds to a strong effect [20]. The Hosmer-Lemeshow test $\left(\mathrm{Chi}^{2}(8)=\right.$ $5.706, p=0.680$ ) indicates a good predictive power.

While all factors showed a significant influence in the univariate regression models, only work experience OR $1.05(95 \% \mathrm{Cl} 1.001-1.104)$ continuously showed a significant influence in the multivariate model (Table 4).

Table 4

Multivariate logistic regression analysis of influencing factors on PIP-ARena $(n=142)$

\begin{tabular}{|llllllll|}
\hline Variables & B & SE & Wald & df & P value & OR & Cl 95\% \\
\hline Experience year & 0.05 & 0.03 & 3.96 & 1 & 0.046 & 1.05 & $1.001-1.104$ \\
\hline PCN environment & 0.45 & 0.29 & 2.41 & 1 & 0.121 & 1.57 & $0.889-2.763$ \\
\hline Structural conditions & 0.14 & 0.29 & 0.24 & 1 & 0.626 & 1.15 & $0.648-2.054$ \\
\hline Environment of existing processes & 0.14 & 0.29 & 0.25 & 1 & 0.618 & 1.16 & $0.656-2.032$ \\
\hline External defined general conditions & 0.20 & 0.29 & 0.50 & 1 & 0.481 & 1.22 & $0.699-2.136$ \\
\hline Constant & -3.43 & 1.26 & 7.46 & 1 & 0.006 & 0.03 & \\
\hline $\begin{array}{l}\text { PIP-ARena = perceived impact of participation in the ARena project on the decision-making regarding } \\
\text { antibiotic prescribing; PCN = primary care network }\end{array}$
\end{tabular}

In order to take the nested data structure into account, the ICC was calculated and identified at 0.22. Due to the small number of clusters and the small number of cases within the clusters, the risk of bias in the estimations of effects must be taken into account.

\section{Discussion}


Nearly half of primary care physicians reported to have perceived an impact on their decision-making regarding antibiotic prescribing which was attributed to participation in the ARena project. With increasing work experience, the likelihood that participation in the ARena project had an influence on antibiotic prescribing decision-making increased. Other factors, such as the PCN environment, structural conditions, environment of existing processes, or externally defined general conditions showed bivariately a significant positive influence in the bivariate logistic regression analysis. None of these effects were confirmed in the multivariate logistic regression model. This might be due to the small sample size and the number of predictive factors and have resulted in a lack of statistical power to detect effects. Other contextual factors such as practice size or practice area did not have a statistically significant influence. The intervention arm affiliation did not have a statistically significant influence on decision-making on antibiotic prescribing due to participation in the ARena project either.

\section{Comparison to previous research}

Prior research investigated the influence of work experience on antibiotic prescribing decisions with heterogeneous findings. In two international interview studies [21, 22], physicians reported a perceived influence of work experience on their antibiotic prescribing decision. Furthermore, a Canadian study that evaluated linked administrative health care data found that primary care physicians who were mid-career or more advanced in their career were more likely to prescribe antibiotics than physicians with less work experience [23]. However, other results indicated no influence of work experience on antibiotic prescribing decisions [24]. These different findings indicate closer examination in further efforts. According to the TPB, one possible explanation for the significant positive influence of work experience in this study could be a well perceived behavioral control and attitude towards a sustainable use of antibiotics. This may be due to many years of experience in dealing with antibiotic prescribing and to knowledge about antibiotics and AMR. A significant lack of knowledge about antibiotics and AMR, has been reported before, particularly in the group of physicians with little work experience and potentially paired with uncertainty in decision-making regarding antibiotic prescriptions [25]. This might indicate that with increasing work experience and thus accumulated reference points, physicians' confidence in the ability to perform the desired behavior might rise [11]. On the other hand, the effect could also support the assumption of the project's implementation strategy, which specifically targeted knowledge deficits and awareness regarding antibiotics and AMR. Research shows that younger physicians might more likely be guidelineoriented than more experienced physicians [26], who may be less current with evidence and guidelinebased care than younger physicians [25]. The effect measured in this study could thus be explained by the notion that knowledge-transmitting intervention measures are more likely to have an impact on more experienced physicians than on younger physicians, who generally are more likely to take guidelineoriented prescribing decisions based on current evidence. This assumption should be further investigated in future research.

The results of this study suggested that PCNs were a supportive environment in the ARena project. In the bivariate regression, a positive influence on decision-making in antibiotic prescribing was shown. A qualitative study within the process evaluation of ARena pointed in the same direction [19]: Physicians 
described PCNs as supportive with regard to exchange (beliefs, ideas and experiences), management, and the implementation of new routines in practice, which can influence decision-making on antibiotic prescribing $[19,27]$. Research further showed that PCNs can act as effective drivers of innovation and quality improvement, especially when communication and support functioned well within the networks $[19,28,29]$. Being part of a PCN can thus support the adoption of specific behaviors and beliefs $[13,19]$. With regards to the TPB, PCNs as a contextual factor might have had a strong influence on both social norms and attitudes towards a behavior through social interactions and relationships. The analysis showed that physicians in arm II rated the PCN environment more supportive than physicians in arm I and/or III. This could be due to the implementation strategy since in arm II, medical assistants were strongly involved to support the physicians. This intervention may have been seen as an additional supportive factor and might have had a reinforcing effect on the perception of PCNs as a supportive environment in arm II.

When interpreting results, it should be considered that PCN member physicians might show stronger tendencies to innovation and development than physicians who are not part of a PCN $[13,19]$. The data analyzed in this study were generated in the process evaluation carried out alongside ARena. Therefore, comparisons with standard care (no intervention) could not be performed. Participants might have given socially desirable answers on the survey questionnaires. However, potential biases due to the clustered structures in PCNs were accounted for by the MLA where effect sizes marginally differed from the results in the multivariate logistic regression analysis and were statistically unremarkable. An additional table file shows this in more detail (see Additional file 5). Due to this, it was decided to present the results of the multivariate logistic regression in detail for better comprehensibility. However, for further interpretation and discussion of the results, it should be considered that there was a slight cluster effect.

Few reference studies were found which also address general conditions of medical practices. For example, some studies showed that communication and the organisational model have an influence on antibiotic prescribing $[24,30,31]$. However, the results of the bivariate regression in this study show that specific contextual factors such as structural conditions, environment of existing processes, or externally defined general conditions had an influence on participation in the project and influenced decisionmaking on antibiotic prescribing. Due to the mean score variables used in this analysis, a differentiated consideration of the domains is not possible. Nevertheless, the results indicate that such general conditions may have an effect regarding antibiotic prescribing decisions and might be a facilitating or inhibiting factor in an implementation process. As these factors are highly individualized at physician level, practice level, or health system level, it is difficult to compare different study samples at all levels or generalize results. Further research is needed regarding such contextual factors to analyze their impact in detail. In the TPB, Ajzen [11] described the influence of similar conditions such as legal requirements, availability of resources, or cooperations on so-called actual behavioral control. Behavioral control and motivation to behave can both significantly influence the intention and, consequently, decision-making [11]. 
While one study identified a significant influence of practice area on antibiotic prescribing [15], this study found no influence. Furthermore, a study showed that physicians who treated a high number of patients were more likely to prescribe antibiotics [23]. This cannot be confirmed by this study either. In total, it can be seen that general conditions of the medical practice, such as the practice size or practice area, as well as specific conditions as described above, were hardly considered or mentioned in research before [32].

No effects could be attributed to the affiliation to the intervention arm. This could be due to the fact that all groups largely received the same intervention components and that the effects of the additional intervention components in arms II and III, in addition to a small number of cases, were not statistically strong enough. Also, in this analysis, only the assignment to the intervention arm was used as a predictor. More detailed analyses on the uptake of the individual intervention components and their possible influence on physicians' perception of antibiotic prescribing are therefore needed.

Overall, previous studies which explored contextual influencing factors were mainly qualitative. Statistical correlations in the specific context of decision-making regarding the prescription of antibiotics were only examined to a limited extent. This study therefore contributes to the identification of statistical correlations regarding this topic as a first of its kind. As described in the introduction, the description and differentiation of the concept of context is complex. In the $\mathrm{CICl}$, context is differentiated into seven domains [10]. In this study, the predictive factors were classified into all contextual domains related to individual factors and the intervention arm allocation in order to examine their influence and relationships in terms of the $\mathrm{CICl}$. Due to the number of cases, only a few numbers of factors could be analyzed. The large number of contextual factors given the small number of cases can be best considered indicative of relationships and influences only. However, investigating to which extend they jointly influenced decisionmaking in antibiotic prescribing was limited. Large-scale studies are needed to sufficiently investigate a diverse environment around physicians' practices in intervention studies. By doing so, effects between context and $\mathrm{CMCl}$ could be identified and contextual factors in the sense of the $\mathrm{CICl}$ could be covered as broadly as possible.

\section{Strengths And Limitations}

Several strengths and limitations must be considered when interpreting the results of this study. One strength of this study was the application of the STROBE-Statement which granted methods and results to be transparent and comprehensible.

The applied questionnaire was not validated but theory-based in conceptualization. However, it can be assumed that all questions were well understood since the number of missing values was small and they were completely at random. Another strength of the study was the high response rate. Nevertheless, the number of cases was statistically too small to conduct a comprehensive analysis of all possible contextual factors identified in theory [14]. The inclusion of predictive variables was thus limited in multivariate regression analysis and only significant factors of the bivariate regression analysis were included. Given the relatively small sample size and a potential lack of statistical power, the number of 
included factors in the multivariate regression analysis might have reduced the degrees of freedom so that effects were not detected. The study focused on the physicians' perceived impact on decisionmaking regarding antibiotic prescribing through their participation in the ARena project. This is a selfreported impact, which may contain an over- as well as an underestimation of individual performance. In additions, it was not possible to link the results of the process evaluation to the actual prescribing rate from the intervention study. Therefore, no statement can be made about the influence of the contextual factors on actual prescribing behavior.

Finally, the generalizability of the results of this study is limited. On the one hand, the participants' age and work experience might limit the transferability of results to younger primary care physicians. On the other hand, the study setting in PCNs must be considered when transferring results. The nested data cannot exclude the possibility that socially desirable responses were given. In addition, a potential selection bias might be present in participants' pre-existing motivation to reduce antibiotic use. Furthermore, the contextual factors used in this study are specific to the German health care system. Thus, the results cannot simply be transferred to practitioners who do not participate in a PCN as well as to non-German health systems.

\section{Conclusion}

This study showed that primary care physicians perceived an influence on their decision-making on antibiotic prescribing through participation in the ARena project. In particular, longer work experience was shown to be a significant influential factor. This might indicate that knowledge transfer interventions promoting rational prescribing of antibiotics might be successful when particularly more experienced physicians are targeted.

Contextual factors such as PCN environment, structural conditions, environment of existing processes in the medical practice, and externally defined general conditions also showed to have an influence. An effective synergy of contextual factors regarding decision-making was not observed. Further comparable analyses with larger case numbers could comprehensively examine contextual factors on antibiotic prescribing decision-making. This study contributes to the understanding of contextual factors, their influence on prescribing decision-making, and their role in an implementation strategy.

\section{Abbreviations}

AMR: Antimicrobial Resistance; ARena: Sustainable reduction of antibiotic-induced antimicrobial resistance; $\mathrm{Cl}$ : Confidence Interval; $\mathrm{ClCl}$ : The Context and Implementation of Complex Interventions; $\mathrm{CMCl}$ : Complex Multiple Component Interventions; DDD: Defined Daily Dose; GP: General Practitioner; ICC: IntraCluster Correlation Coefficient; MA: Medical Assistant; MLA: Multilevel Analysis; OR: Odds Ratio; PCN: Primary Care Network; SD: Standard Deviation; TPB: Theory of Planned Behaviour

\section{Declarations}




\section{Ethics approval and consent to participate}

Ethical clearance for the ARena study was obtained from the Ethics Committee of the Medical Faculty of Heidelberg University (reference number S-353/2017). All participants in the process evaluation gave written informed consent. Confidentiality and anonymity were ensured throughout the study [6].

\section{Consent for publication}

Not applicable.

\section{Availability of data and materials}

The datasets used and analysed during the current study are available from the corresponding author on reasonable request. An overview of all relevant survey items for this analysis can be found in Additional file 1.

\section{Competing interests}

The authors declare that they have no competing interests.

\section{Funding}

The ARena study received funding from the Innovation Committee at the Federal Joint Committee (G-BA), Berlin (01NVF16008). The funder had no role in the design, data-collection, data-analysis, interpretation, or writing of the paper.

\section{Authors' contributions}

AQ drafted and prepared the manuscript. AQ, CA, MW, and RPD contributed to concept and design of this study. RPD, MW, and MK (non-author contributor) collaborated on the construction of the survey questionnaires and collected the data. AQ analyzed and interpreted the data for this study. CA, DK (nonauthor contributor), MW, and RPD provided methodical guidance. All authors provided substantial comments and approved the final version of the manuscript.

AQ - Annika Queder; CA - Christine Arnold; MW - Michel Wensing; RPD - Regina Poß-Doering

Non-author contributors: DK - Dorothea Kronsteiner; MK - Martina Kamradt

\section{Acknowledgements}

The authors would like to thank the participants in the ARena project for their contribution, the staff at aQua Institute, Goettingen, for their overall support in ARena, and Dorothea Kronsteiner, Department of Medical Biometry, Institute of Medical Biometry and Informatics, University Hospital Heidelberg, for statistical advice and methodical guidance during the process of data analysis. 


\section{References}

1. Bundesministerium für Gesundheit. DART 2020: Antibiotika-Resistenzen bekämpfen zum Wohl von Mensch und Tier [Internet]; 2015 [cited 2021 May 26]. Available from: URL: https://www.bundesgesundheitsministerium.de/fileadmin/Dateien/3_Downloads/A/AntibiotikaResistenz-Strategie/BMG_DART_Bericht_10_RZ_03_web_neu.pdf.

2. Bell BG, Schellevis F, Stobberingh E, Goossens H, Pringle M. A systematic review and meta-analysis of the effects of antibiotic consumption on antibiotic resistance. BMC Infect Dis 2014; 14:13.

3. Lum EPM, Page K, Whitty JA, Doust J, Graves N. Antibiotic prescribing in primary healthcare: Dominant factors and trade-offs in decision-making. Infection, Disease \& Health 2018; 23(2):74-86.

4. Centre for Disease Prevention and Control. Antimicrobial consumption in the EU/EEA - Annual Epidemiological Report 2019 [Surveillance Report] [Internet]. Stockholm: ECDC; 2020 [cited 2021 May 25]. Available from: URL: https://www.ecdc.europa.eu/sites/default/files/documents/Antimicrobialconsumption-in-the-EU-Annual-Epidemiological-Report-2019.pdf.

5. Kraus EM, Pelzl S, Szecsenyi J, Laux G. Antibiotic prescribing for acute lower respiratory tract infections (LRTI) - guideline adherence in the German primary care setting: An analysis of routine data. PLoS ONE 2017; 12(3):e0174584.

6. Kamradt M, Kaufmann-Kolle P, Andres E, Brand T, Klingenberg A, Glassen K et al. Sustainable reduction of antibiotic-induced antimicrobial resistance (ARena) in German ambulatory care: study protocol of a cluster randomised trial. Implement Sci 2018; 13(1):23.

7. Schrappe M, Pfaff M. Versorgungsforschung vor neuen Herausforderungen: Konsequenzen für Definition und Konzept. Gesundheitswesen 2016 [cited 2021 May 26]; 78(11):689-94. Available from: URL: https://www.thieme-connect.com/products/ejournals/pdf/10.1055/s-0042-116230.pdf? update=true.

8. Geraedts M, Drösler SE, Döbler K, Eberlein-Gonska M, Heller G, Kuske S et al. DNVF-Memorandum III "Methoden für die Versorgungsforschung", Teil 3: Methoden der Qualitäts- und Patientensicherheitsforschung. Gesundheitswesen 2017; 79(10):e95-e124.

9. Pfadenhauer LM, Mozygemba K, Gerhardus A, Hofmann B, Booth A, Lysdahl KB et al. Context and implementation: A concept analysis towards conceptual maturity. Z Evid Fortbild Qual Gesundhwes 2015; 109(2):103-14.

10. Pfadenhauer LM, Gerhardus A, Mozygemba K, Lysdahl KB, Booth A, Hofmann B et al. Making sense of complexity in context and implementation: the Context and Implementation of Complex Interventions (CICl) framework. Implement Sci 2017; 12(1):21.

11. Ajzen I. The theory of planned behavior. Organizational Behavior and Human Decision Processes 1991; 50(2):179-211.

12. Elm E von, Altman DG, Egger M, Pocock SJ, Gøtzsche PC, Vandenbroucke JP. Das Strengthening the Reporting of Observational Studies in Epidemiology (STROBE-) Statement. Notfall Rettungsmed 2008; 11(4):260-5. 
13. Poss-Doering R, Kühn L, Kamradt M, Stürmlinger A, Glassen K, Andres E et al. Fostering Appropriate Antibiotic Use in a Complex Intervention: Mixed-Methods Process Evaluation Alongside the ClusterRandomized Trial ARena. Antibiotics (Basel) 2020; 9(12).

14. Hosmer DW, Lemeshow S. Applied Logistic Regression. Hoboken, NJ, USA: John Wiley \& Sons, Inc; 2000.

15. Liu C, Liu C, Wang D, Zhang X. Intrinsic and external determinants of antibiotic prescribing: a multilevel path analysis of primary care prescriptions in Hubei, China. Antimicrob Resist Infect Control 2019; 8:132.

16. Ansmann L, Kuhr K, Kowalski C. Mehrebenenanalysen in der organisationsbezogenen Versorgungsforschung - Nutzen, Voraussetzungen und Durchführung. Gesundheitswesen 2017; 79(3):203-9.

17. Cohen J, Cohen P, West SG, Aiken LS, editors. Applied Multiple Regression/Correlation Analysis for the Behavioral Sciences. 3rd ed.: Routledge; 2013.

18. Sommet N, Morselli D. Keep Calm and Learn Multilevel Logistic Modeling: A Simplified Three-Step Procedure Using Stata, R, Mplus, and SPSS. International Review of Social Psychology 2017; 30(1):203-18.

19. Poss-Doering R, Kamradt M, Glassen K, Andres E, Kaufmann-Kolle P, Wensing M. Promoting rational antibiotic prescribing for non-complicated infections: understanding social influence in primary care networks in Germany. BMC Fam Pract 2020; 21(1):51.

20. Cohen J. Statistical Power Analysis for the Behavioral Sciences.

21. Paredes P, La Peña M de, Flores-Guerra E, Diaz J, Trostle J. Factors influencing physicians' prescribing behaviour in the treatment of childhood diarrhoea: Knowledge may not be the clue. Social Science \& Medicine 1996; 42(8):1141-53.

22. Mol PGM, Rutten WJMJ, Gans ROB, Degener JE, Haaijer-Ruskamp FM. Adherence barriers to antimicrobial treatment guidelines in teaching hospital, the Netherlands. Emerg Infect Dis 2004; 10(3):522-5.

23. Silverman M, Povitz M, Sontrop JM, Li L, Richard L, Cejic S et al. Antibiotic Prescribing for Nonbacterial Acute Upper Respiratory Infections in Elderly Persons. Ann Intern Med 2017; 166(11):765-74.

24. Paluck E, Katzenstein D, Frankish CJ, Herbert CP, Milner R, Speert D et al. Prescribing practices and attitudes toward giving children antibiotics. Can Fam Physician 2001; 47:521-7.

25. Neugebauer M, Ebert M, Vogelmann R. Mangel an Informationen und deren Bereitstellung am Arbeitsplatz als mögliche Gründe für inkorrekte Antibiotikatherapien in Deutschland. Z Evid Fortbild Qual Gesundhwes 2019; 144-145:35-41.

26. Butzlaff M, Kempkens D, Schnee M, Dieterle WE, Böcken J, Rieger MA. German ambulatory care physicians' perspectives on clinical guidelines - a national survey. BMC Fam Pract 2006; 7:47.

27. Keating NL. Peer Influence and Opportunities for Physician Behavior Change. J Natl Cancer Inst 2017; 109(8). 
28. Gabriel J. Praxisnetze im Wandel - Chancen und Stärken eines Versorgungsmodells. In: Pfannstiel MA, Focke A, Mehlich H, editors. Management von Gesundheitsregionen III: Gesundheitsnetzwerke zur Optimierung der Krankenversorgung durch Kooperation und Vernetzung. Wiesbaden: Springer Gabler; 2017. p. 13-24.

29. Pollack CE, Soulos PR, Herrin J, Xu X, Christakis NA, Forman HP et al. The Impact of Social Contagion on Physician Adoption of Advanced Imaging Tests in Breast Cancer. J Natl Cancer Inst 2017; 109(8).

30. Walker S, McGeer A, Simor AE, Armstrong-Evans, Loeb M. Why are antibiotics prescribed for asymptomatic bacteriuria in institutionalized elderly people?: A qualitative study of physicians' and nurses' perceptions. CMAJ 2000; 163(3):273-7.

31. Mangione-Smith R, Elliott MN, Stivers T, McDonald LL, Heritage J. Ruling out the need for antibiotics: are we sending the right message? Arch Pediatr Adolesc Med 2006; 160(9):945-52.

32. Teixeira Rodrigues A, Roque F, Falcão A, Figueiras A, Herdeiro MT. Understanding physician antibiotic prescribing behaviour: a systematic review of qualitative studies. Int J Antimicrob Agents 2013; 41(3):203-12.

\section{Supplementary Files}

This is a list of supplementary files associated with this preprint. Click to download.

- Additionalfile1TranslatedOverviewrelevantquestions.pdf.pdf

- Additionalfile2OverviewandReliabilitymeanscorevar.pdf

- Additionalfile3Missingvalueanalysis.pdf.pdf

- Additionalfile4Resultsinterventionarmcomparison.pdf.docx

- Additionalfile5Resultsmultilevelanalysis.pdf.pdf 